\title{
Diastereoselective Addition of Organocerium(III) Reagents Derived from 3-Substituted Propargyl Bromides to Aldehydes ${ }^{1}$
}

\author{
Ulrich Groth, * Christian Kesenheimer, Jürgen Neidhöfer \\ Fachbereich Chemie, Universität Konstanz, Universitätsstrasse 11, Postfach M-720, 78457 Konstanz, Germany \\ Fax +49(7531)884155; E-mail: ulrich.groth@uni-konstanz.de
}

\begin{abstract}
Various cerium allenyl reagents were generated by transmetallation of allenyl Grignard compounds with $\mathrm{CeCl}_{3}$ and subsequent conversion into homopropargylic alcohols by addition to various aliphatic and aromatic aldehydes. The $\alpha$-acetylenic alcohols were obtained with regioselectivities and diastereoselectivities up to 98\% de in favor of the threo-diastereomers.
\end{abstract}

Key words: lanthanides, organometallic reagents, addition reactions, diastereoselectivity, regioselectivity

The importance of allenic anions in synthetic organic chemistry arises from their utility in $\mathrm{C}-\mathrm{C}$ bond forming reactions such as additions to carbonyl groups. ${ }^{2}$ The resulting homopropargylic alcohols are key intermediates in the synthesis of $\gamma$-butyrolactones ${ }^{3}$ and polyketide natural products. ${ }^{4}$ As ambident nucleophiles allenic anions add to carbonyl groups giving rise to two products, $\beta$-acetylenic and $\alpha$-allenic alcohols. ${ }^{5}$ Furthermore, the products can be formed as mixtures of two diastereomers. Thus, to achieve a selective reaction, it is necessary to control the regioand stereochemistry at the same time. Structure and reactivities of the ambident anions are highly dependent on the nature of the counter cation (Scheme 1). ${ }^{6}$
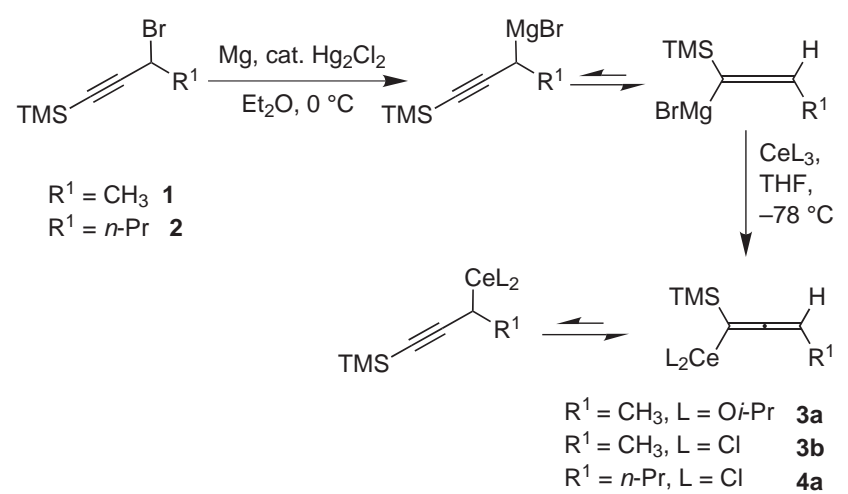

Scheme 1 Generation of the organocerium(III) allenyl compounds

In the course of our studies in natural product synthesis of compounds such as ergosterol ${ }^{7}$ and its 4-substituted derivatives we examined the influence of different cerium(III) species. ${ }^{8}$ After transmetallation of the Grignard reagent prepared from 1-(trimethylsilyl)propargyl bromide with
$\mathrm{CeCl}_{3}$ or $\mathrm{Ce}(\mathrm{O} i-\mathrm{Pr})_{3}$, no change in the regioselectivity but an enormous improvement of the diastereoselectivity of the carbonyl addition reaction was observed in comparison to the Grignard reagent.

Because of the outstanding diastereoselectivities often reached with organocerium compounds in addition reactions to carbonyl groups, ${ }^{9}$ we investigated the suitability of these organometallics for a diastereoselective synthesis of $\beta$-acetylenic alcohols (Scheme 2, Table 2).

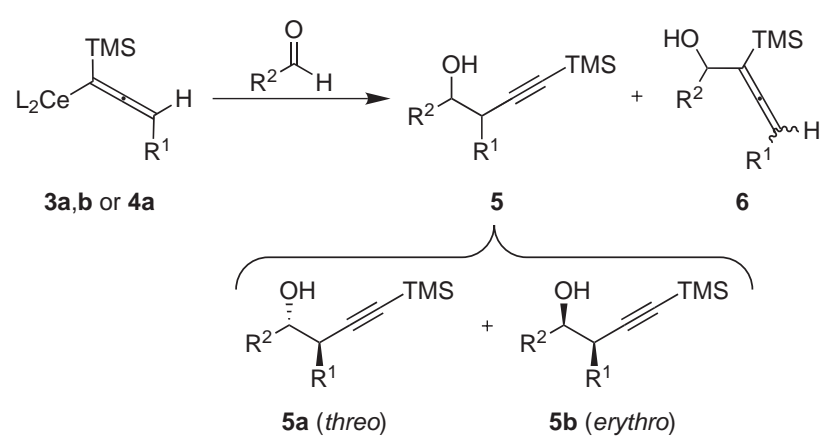

Scheme 2 Reaction of organocerium(III)allenyls with aldehydes

Based on earlier results ${ }^{8}$ we chose $\mathrm{Ce}(\mathrm{O} i-\mathrm{Pr})_{3}$ as cerium reagent, whereas benzaldehyde and 3-bromo-1-(trimethylsilyl)but-1-yne (1) ${ }^{10}$ were selected as model compounds to optimize the reaction conditions. First, we decided to study the effect of the reaction temperature on yield and selectivity of the addition. The results are shown in Table 1.

Addition of the benzaldehyde to the organocerium reagent at $-78{ }^{\circ} \mathrm{C}$ followed by warming to room temperature within 16 hours afforded the homopropargylic alcohols $\mathbf{5 a}$ and $\mathbf{5 b}$ in $50 \%$ yield with a regioisomeric ratio of $>98: 2$ and a diastereomeric ratio of 66:34 (entry 1). In addition, benzyl alcohol was formed among other by-products. The same reaction at $0{ }^{\circ} \mathrm{C}$ followed by warming to room temperature within two hours afforded $\mathbf{5 a}$ and $\mathbf{5 b}$ in an overall yield of $72 \%$ with unchanged regioselectivity and a diastereomeric ratio of 72:28. Only traces of benzyl alcohol could be found. Using these reaction conditions, we studied as next the effect of the cerium reagent.

Applying $\mathrm{CeCl}_{3}$ for synthesizing the cerium allenyl reagent out of the Grignard reagent at $0{ }^{\circ} \mathrm{C}$ gave similar results (Table 1, entry 3$)$ as $\mathrm{Ce}(\mathrm{O} i-\mathrm{Pr})_{3}$ under the reaction conditions A with benzaldehyde. Best results were obtained by employing $\mathrm{CeCl}_{3}$ under reaction conditions $\mathrm{B}$ 
Table 1 Addition of Cerium Allenyl Compounds $\mathbf{3 a}$ and $\mathbf{3 b}$ to Benzaldehyde

\begin{tabular}{llllll}
\hline Entry & Reaction conditions $^{\mathbf{a}}$ & Cerium reagent & Yield $(\%)$ of $\mathbf{5}+\mathbf{6}$ & $\mathbf{5 : 6}$ & $\mathbf{5 a}: \mathbf{5 b}$ \\
\hline 1 & $\mathrm{~A}$ & $\mathrm{Ce}(\mathrm{O} i-\mathrm{Pr})_{3} \mathbf{3 a}$ & 50 & $>98: 2$ & $66: 34$ \\
2 & $\mathrm{~B}$ & $\mathrm{Ce}(\mathrm{O} i-\mathrm{Pr})_{3} \mathbf{3 a}$ & 72 & $>98: 2$ & $72: 28$ \\
3 & $\mathrm{CeCl}_{3} \mathbf{3 b}$ & 51 & $>98: 2$ & $67: 33$ \\
4 & $\mathrm{~A}$ & $\mathrm{CeCl}_{3} \mathbf{3 b}$ & 90 & $>98: 2$ & $70: 30$ \\
\hline
\end{tabular}

${ }^{a} \mathrm{~A}:-78{ }^{\circ} \mathrm{C}$ to r.t., $16 \mathrm{~h} ; \mathrm{B}: 0{ }^{\circ} \mathrm{C}$ to r.t., $2 \mathrm{~h}$.

(Table 1, entry 4). The overall yield of $\mathbf{5 a}$ and $\mathbf{5 b}$ increased to $90 \%$ with almost the same regio- and diastereoselectivity as in entries 1,2 and 3 . Our results with other aldehydes are summarized in Table 2.

The overall yield of 5 and $\mathbf{6}$ ranges from $56 \%$ to $90 \%$, whereas addition to benzaldehyde, $p$-anisaldehyde and pivalaldehyde afforded predominantly the respective homopropargylic alcohols $\mathbf{5 a}$ and $\mathbf{5 b}$ with a regioselectivity of $>98: 2$. Lower regioselectivities were observed when cyclohexylcarbaldehyde (entries 3, 8 and 13) and acetaldehyde (entries 5, 10 and 15) were employed. The influence of the aldehyde on the regioselectivity of the addition has already been observed before. However, the reason for this phenomenon still remains unkown. ${ }^{5}$

The homopropargylic alcohols 5a were obtained with threolerythro ratios ranging from $65: 35$ to $>98: 2$. Slightly higher diastereoselectivities were obtained in reactions of the organocerium reagent $\mathbf{4 a}$ derived from 3-bromo-1(trimethylsilyl)hex-1-yne $(2)^{11}$ (entries 11-15). In accordance with former studies, moderate diastereomeric ratios were observed by employing benzaldehyde (entries 1, 6 and 11) and $p$-anisaldehyde (entries 2,7 and 12). ${ }^{6}$ On the other hand, the high diasteroselectivities of 98:2 observed in the addition reactions to pivalaldehyde are remarkable (entries 4, 9 and 14).

In summary, we have presented a useful method for the synthesis threo-homopropargylic alcohols. An enantioselective synthesis of these compounds using chiral modified cerium reagents is under current investigation.

\section{Typical Experimental Procedure}

All reactions were carried out under an argon atmosphere using Schlenck techniques. Moisture and oxidation sensitive compounds were stored in a glove box.

Table 2 Addition of Cerium Allenyl Compounds 3a, $\mathbf{b}$ and $4 \mathbf{a}$ to Different Aldehydes

\begin{tabular}{|c|c|c|c|c|c|}
\hline Entry & Bromide $\mathrm{R}^{1}$ & Aldehyde $\mathrm{R}^{2}$ & Yield $(\%)^{\mathrm{b}}$ of $\mathbf{5}+\mathbf{6}$ & $5: 6$ & $5 \mathbf{a}: 5 \mathbf{b}$ \\
\hline 1 & $\mathrm{Me}$ & $\mathrm{Ph}$ & 90 & $>98: 2$ & $70: 30$ \\
\hline 2 & $\mathrm{Me}$ & $4-\mathrm{MeOC}_{6} \mathrm{H}_{4}$ & 90 & $>98: 2$ & $65: 35$ \\
\hline 3 & $\mathrm{Me}$ & Cyclohexyl & 80 & $92: 8$ & $80: 20$ \\
\hline 4 & $\mathrm{Me}$ & $t$-Bu & 74 & $>98: 2$ & $98: 2$ \\
\hline 5 & $\mathrm{Me}$ & $\mathrm{Me}$ & 85 & $89: 11$ & $81: 19$ \\
\hline 6 & $\mathrm{Me}^{\mathrm{a}}$ & $\mathrm{Ph}$ & 72 & $>98: 2$ & $72: 28$ \\
\hline 7 & $\mathrm{Me}^{\mathrm{a}}$ & $4-\mathrm{MeOC}_{6} \mathrm{H}_{4}$ & 70 & $>98: 2$ & $68: 32$ \\
\hline 8 & $\mathrm{Me}^{\mathrm{a}}$ & Cyclohexyl & 66 & $92: 8$ & $85: 15$ \\
\hline 9 & $\mathrm{Me}^{\mathrm{a}}$ & $t-\mathrm{Bu}$ & 56 & $>98: 2$ & $98: 2$ \\
\hline 10 & $\mathrm{Me}^{\mathrm{a}}$ & $\mathrm{Me}$ & 64 & $86: 14$ & $79: 21$ \\
\hline 11 & $n$-Pr & $\mathrm{Ph}$ & 85 & $>98: 2$ & $72: 28$ \\
\hline 12 & $n$-Pr & 4- $\mathrm{MeOC}_{6} \mathrm{H}_{4}$ & 84 & $>98: 2$ & $68: 32$ \\
\hline 13 & $n$-Pr & Cyclohexyl & 72 & $90: 10$ & $82: 18$ \\
\hline 14 & $n$-Pr & $t$-Bu & 56 & $>98: 2$ & $>98: 2$ \\
\hline 15 & $n$-Pr & $\mathrm{Me}$ & 84 & $86: 14$ & $85: 15$ \\
\hline
\end{tabular}

${ }^{\mathrm{a}} \mathrm{Ce}(\mathrm{O} i-\mathrm{Pr})_{3}$ was used instead of $\mathrm{CeCl}_{3}$.

${ }^{\mathrm{b}}$ Reaction conditions: $0{ }^{\circ} \mathrm{C}$ to r.t., $2 \mathrm{~h}$; isolated yield. 
$\mathrm{Mg}(0.61 \mathrm{~g}, 25.0 \mathrm{mmol})$ and $\mathrm{Hg}_{2} \mathrm{Cl}_{2}(5.0 \mathrm{mg}, 0.01 \mathrm{mmol})$ were suspended in $\mathrm{Et}_{2} \mathrm{O}(20 \mathrm{~mL})$ at r.t. and 1,2-dibromoethane $(0.1 \mathrm{~mL})$ was added. The mixture was stirred for $1 \mathrm{~h}$ and then cooled to $0{ }^{\circ} \mathrm{C}$. Within 1 h compound $1(1.0 \mathrm{~g}, 4.7 \mathrm{mmol})$ or compound $2(1.1 \mathrm{~g}, 4.7$ mmol) was added by use of a syringe pump while the temperature was maintained between $0{ }^{\circ} \mathrm{C}$ and $5^{\circ} \mathrm{C}$. After stirring for an additional hour at r.t. the mixture was added at $0{ }^{\circ} \mathrm{C}$ to a suspension of the cerium(III) compound $(4.7 \mathrm{mmol})$ in THF via cannula. The resulting solution was stirred then at $0{ }^{\circ} \mathrm{C}$ for $1 \mathrm{~h}$. Subsequently, 3 mmol of the aldehyde were added and the mixture was allowed to warm up to r.t. within $2 \mathrm{~h}$. The solvent was removed in vacuo $\left(20^{\circ} \mathrm{C}, 15 \mathrm{mbar}\right)$ and the residue was suspended in $\mathrm{Et}_{2} \mathrm{O}(150 \mathrm{~mL})$. After addition of $1 \mathrm{M} \mathrm{HCl}(50 \mathrm{~mL})$, the layers were separated and the aqueous layer was extracted with $\mathrm{Et}_{2} \mathrm{O}(3 \times 50 \mathrm{~mL})$. The combined organic layers were extracted with sat. $\mathrm{NaHCO}_{3}$ solution $(50$ $\mathrm{mL})$ and brine $(50 \mathrm{~mL})$ and dried over $\mathrm{Na}_{2} \mathrm{SO}_{4}$. The solvent was then removed in vacuo $\left(20^{\circ} \mathrm{C}, 15 \mathrm{mbar}\right)$ again. The crude products were purified by flash chromatography on silica gel (eluent: PE$\mathrm{Et}_{2} \mathrm{O}$, depending on the product). The regioisomeric and diastereomeric ratios were determined by GCMS analysis of the crude products.

\section{Analytical Data \\ threo-2-Methyl-1-phenyl-4-trimethylsilylbut-3-yn-1-ol ${ }^{6}$}

${ }^{1} \mathrm{H} \mathrm{NMR}\left(250 \mathrm{MHz}, \mathrm{CDCl}_{3}\right): \delta=0.17$ (s, $\left.9 \mathrm{H}, \mathrm{TMS}\right), 1.18(\mathrm{~d}, 3 \mathrm{H}$, $\mathrm{CH}_{3}, J=7.0 \mathrm{~Hz}$ ), $2.24(\mathrm{~d}, 1 \mathrm{H}, \mathrm{OH}, J=3.5 \mathrm{~Hz}), 2.87$ (quint, $1 \mathrm{H}, \mathrm{H}-$ $2, J=7.0 \mathrm{~Hz}), 4.47$ (dd, $1 \mathrm{H}, \mathrm{H}-1, J=7.0,3.5 \mathrm{~Hz}), 7.30-7.43(\mathrm{~m}, 5$ $\mathrm{H}, \mathrm{Ph})$.

erythro-2-Methyl-1-phenyl-4-trimethylsilylbut-3-yn-1-ol ${ }^{6}$ ${ }^{1} \mathrm{H}$ NMR $\left(250 \mathrm{MHz}, \mathrm{CDCl}_{3}\right): \delta=0.12(\mathrm{~s}, 9 \mathrm{H}, \mathrm{TMS}), 1.07(\mathrm{~d}, 3 \mathrm{H}$, $\left.\mathrm{CH}_{3}, J=7.0 \mathrm{~Hz}\right), 2.50(\mathrm{~d}, 1 \mathrm{H}, \mathrm{OH}, J=3.5 \mathrm{~Hz}), 2.79(\mathrm{~m}, 1 \mathrm{H}, \mathrm{H}-2)$, $4.76(\mathrm{dd}, 1 \mathrm{H}, \mathrm{H}-1, J=6.5,3.5 \mathrm{~Hz}), 7.28-7.41(\mathrm{~m}, 5 \mathrm{H}, \mathrm{Ph})$.

\section{threo-1-(4-Methoxyphenyl)-2-methyl-4-trimethylsilyl- but-3-yn-1-ol}

${ }^{1} \mathrm{H}$ NMR $\left(250 \mathrm{MHz}, \mathrm{CDCl}_{3}\right): \delta=0.18$ (s, $\left.9 \mathrm{H}, \mathrm{TMS}\right), 1.04$ (d, $3 \mathrm{H}$, $\mathrm{CH}_{3}, J=7.0 \mathrm{~Hz}$ ), $2.61(\mathrm{~d}, 1 \mathrm{H}, \mathrm{OH}, J=3.0 \mathrm{~Hz}$ ), 2.67-2.75 (quint, 1 $\mathrm{H}, \mathrm{H}-2, J=7.0 \mathrm{~Hz}), 3.80\left(\mathrm{~s}, 3 \mathrm{H}, \mathrm{OCH}_{3}\right), 4.20(\mathrm{dd}, 1 \mathrm{H}, \mathrm{H}-1, J=7.0$, $3.0 \mathrm{~Hz}), 6.87\left(\mathrm{~d}, 2 \mathrm{H}, \mathrm{H}-3^{\prime}\right.$ and $\left.\mathrm{H}^{-} 5^{\prime}, J=9.3 \mathrm{~Hz}\right), 7.28\left(\mathrm{~d}, 2 \mathrm{H}, \mathrm{H}-2^{\prime}\right.$ and $\left.\mathrm{H}-6^{\prime}, J=9.3 \mathrm{~Hz}\right) .{ }^{13} \mathrm{C}$ NMR $\left(62.5 \mathrm{MHz}, \mathrm{CDCl}_{3}\right): \delta=0.08$ (TMS), $17.17\left(\mathrm{C} 2-\mathrm{CH}_{3}\right), 36.54(\mathrm{C}-2), 55.25\left(\mathrm{OCH}_{3}\right), 76.5(\mathrm{C}-1)$, $87.92(\mathrm{C}-4), 107.88(\mathrm{C}-3), 113.6\left(\mathrm{C}-3^{\prime}\right.$ and $\left.\mathrm{C}-5^{\prime}\right), 127.87\left(\mathrm{C}-2^{\prime}\right.$ and C-6'), $133.40\left(\mathrm{C}-1^{\prime}\right), 159.30\left(\mathrm{C}-4^{\prime}\right)$. MS (GCMS): $m / z(\%)=137$ (100), 73 (12). IR (hexane): $2163(\mathrm{C} \equiv \mathrm{C}) \mathrm{cm}^{-1}$. Anal. Calcd for $\mathrm{C}_{15} \mathrm{H}_{22} \mathrm{O}_{2} \mathrm{Si}$ : C, 68.65; H, 8.45. Found: C, 68.75; H, 7.96.

erythro-1-(4-Methoxyphenyl)-2-methyl-4-trimethylsilylbut-3-yn-1-ol

${ }^{1} \mathrm{H}$ NMR (250 MHz, CDCl $)_{3}$ ): $\delta=0.14$ (s, $\left.9 \mathrm{H}, \mathrm{TMS}\right), 1.11$ (d, $3 \mathrm{H}$, $\left.\mathrm{CH}_{3}, J=7.0 \mathrm{~Hz}\right), 2.23(\mathrm{~d}, 1 \mathrm{H}, \mathrm{OH}, J=3.3 \mathrm{~Hz}), 2.75-2.99(\mathrm{~m}, 1 \mathrm{H}$, $\mathrm{H}-2), 3.83\left(\mathrm{~s}, 3 \mathrm{H}, \mathrm{OCH}_{3}\right), 4.68(\mathrm{~m}, 1 \mathrm{H}, \mathrm{H}-1), 6.88\left(\mathrm{~d}, 2 \mathrm{H}, \mathrm{H}-2^{\prime}\right.$ and $\left.\mathrm{H}-6^{\prime}, J=9.3 \mathrm{~Hz}\right), 7.32$ (d, $2 \mathrm{H}, \mathrm{H}-3^{\prime}$ and $\left.\mathrm{H}-5^{\prime}, J=9.3 \mathrm{~Hz}\right) .{ }^{13} \mathrm{C} \mathrm{NMR}$ $\left(62.5 \mathrm{MHz}, \mathrm{CDCl}_{3}\right): \delta=0.05(\mathrm{TMS}), 15.82\left(\mathrm{C} 2_{-}-\mathrm{CH}_{3}\right), 35.45(\mathrm{C}-2)$, $55.26\left(\mathrm{OCH}_{3}\right), 76.23(\mathrm{C}-1), 87.36(\mathrm{C}-4), 108.31(\mathrm{C}-3), 113.35\left(\mathrm{C}-3^{\prime}\right.$ and $\left.\mathrm{C}-5^{\prime}\right), 127.71$ (C-2' and C-6'), $133.54\left(\mathrm{C}-1^{\prime}\right), 159.11\left(\mathrm{C}-4^{\prime}\right)$. GCMS: identical to the threo-compound. IR (hexane): $2165(\mathrm{C} \equiv \mathrm{C})$ $\mathrm{cm}^{-1}$. Anal. Calcd for $\mathrm{C}_{15} \mathrm{H}_{22} \mathrm{O}_{2} \mathrm{Si}: \mathrm{C}, 68.65 ; \mathrm{H}, 8.45$. Found: $\mathrm{C}$, 68.91; H, 8.42.

threo-1-Cyclohexyl-2-methyl-4-trimethylsilylbut-3-yn-1-ol ${ }^{6}$ ${ }^{1} \mathrm{H}$ NMR $\left(250 \mathrm{MHz}, \mathrm{CDCl}_{3}\right): \delta=0.16(\mathrm{~s}, 9 \mathrm{H}, \mathrm{TMS}), 0.93-2.05(\mathrm{~m}$, $15 \mathrm{H}$, cyclohexyl, $\left.\mathrm{CH}_{3}, \mathrm{OH}\right), 2.73(\mathrm{dq}, 1 \mathrm{H}, \mathrm{H}-2, J=7.0,4.8 \mathrm{~Hz})$, 3.07 (dq, $1 \mathrm{H}, \mathrm{H}-1, J=7.0,4.8 \mathrm{~Hz}$ ).
erythro-1-Cyclohexyl-2-methyl-4-trimethylsilylbut-3-yn-1-ol ${ }^{\mathbf{6}}$ ${ }^{1} \mathrm{H}$ NMR $\left(250 \mathrm{MHz}, \mathrm{CDCl}_{3}\right): \delta=0.15$ (s, $\left.9 \mathrm{H}, \mathrm{TMS}\right), 0.88-2.00(\mathrm{~m}$, $15 \mathrm{H}$, cyclohexyl, $\left.\mathrm{CH}_{3}, \mathrm{OH}\right), 2.66(\mathrm{dq}, 1 \mathrm{H}, \mathrm{H}-2, J=10.0,7.0 \mathrm{~Hz})$, $3.33(\mathrm{dq}, 1 \mathrm{H}, \mathrm{H}-1, J=10.0,3.8 \mathrm{~Hz})$.

\section{threo-2,2,4-Trimethyl-6-trimethylsilylhex-5-yn-3-ol}

${ }^{1} \mathrm{H}$ NMR $\left(250 \mathrm{MHz}, \mathrm{CDCl}_{3}\right.$ ): $\delta=0.14$ (s, $\left.9 \mathrm{H}, \mathrm{TMS}\right), 0.95$ (s, $9 \mathrm{H}$, $t$-Bu), $1.29\left(\mathrm{~d}, 3 \mathrm{H}, \mathrm{CH}_{3}, J=7.0 \mathrm{~Hz}\right), 1.96(\mathrm{~d}, 1 \mathrm{H}, \mathrm{OH}, J=10.7 \mathrm{~Hz})$, $2.82(\mathrm{dq}, 1 \mathrm{H}, \mathrm{H}-4, J=10.7,7.0 \mathrm{~Hz}), 2.97$ (dd, $1 \mathrm{H}, \mathrm{H}-3, J=10.7$, $1.4 \mathrm{~Hz}) .{ }^{13} \mathrm{C} \mathrm{NMR}\left(62.5 \mathrm{MHz}, \mathrm{CDCl}_{3}\right): \delta=-0.03(-\mathrm{TMS}), 21.24$ $\left.\left(-\mathrm{CH}_{3}\right), 26.32\left[-\mathrm{C}\left(\mathrm{CH}_{3}\right)_{3}\right], 29.97(\mathrm{C}-4), 36.11\left[\mathrm{C}^{\left(\mathrm{CH}_{3}\right.}\right)_{3}\right], 81.41(\mathrm{C}-$ 3), $89.68(\mathrm{C}-6), 107.44(\mathrm{C}-5)$. GCMS: $m / z(\%)=212(<1)\left[\mathrm{M}^{+}\right], 197$ (<1) $\left[\mathrm{M}-\mathrm{CH}_{3}\right]^{+}, 159(21), 126(30), 73$ (100) [TMS $]^{+}$. Anal. Calcd for $\mathrm{C}_{12} \mathrm{H}_{24} \mathrm{OSi}$ : C, 67.86; H, 11.39. Found: $\mathrm{C}, 66.68 ; \mathrm{H}, 10.82$.

erythro-2,2,4-Trimethyl-6-trimethylsilylhex-5-yn-3-ol

${ }^{1} \mathrm{H}$ NMR $\left(250 \mathrm{MHz}, \mathrm{CDCl}_{3}\right.$ ): $\delta=0.13$ (s, $\left.9 \mathrm{H}, \mathrm{TMS}\right), 0.99$ (s, $9 \mathrm{H}$, $t$ - $\mathrm{Bu}), 1.23\left(\mathrm{~d}, 3 \mathrm{H}, \mathrm{CH}_{3}, J=7.0 \mathrm{~Hz}\right), 1.75(\mathrm{~d}, 1 \mathrm{H}, \mathrm{OH}, J=4.5 \mathrm{~Hz})$, 2.69 (dq, $1 \mathrm{H}, \mathrm{H}-4, J=7.0,5.4 \mathrm{~Hz}), 3.38$ (dd, $1 \mathrm{H}, \mathrm{H}-3, J=5.4,4.5$ $\mathrm{Hz}) .{ }^{13} \mathrm{C} \mathrm{NMR}\left(62.5 \mathrm{MHz}, \mathrm{CDCl}_{3}\right): \delta=-0.03$ (TMS), $20.94\left(\mathrm{CH}_{3}\right)$, $26.22\left[\mathrm{C}\left(\mathrm{CH}_{3}\right)_{3}\right], 30.05(\mathrm{C}-4), 36.11\left[\mathrm{C}\left(\mathrm{CH}_{3}\right)_{3}\right], 81.41(\mathrm{C}-3), 89.68$ (C-6), 107.44 (C-5). GCMS: identical to the threo-compound. Anal. Calcd for $\mathrm{C}_{12} \mathrm{H}_{24} \mathrm{OSi}$ : C, 67.86; H, 11.39. Found: C, 66.96; H, 10.28.

\section{threo-3-Methyl-5-trimethylsilylpent-4-yn-2-ol}

${ }^{1} \mathrm{H}$ NMR (250 MHz, $\mathrm{CDCl}_{3}$ ): $\delta=0.15$ (s, $\left.9 \mathrm{H}, \mathrm{TMS}\right), 1.18$ (d, $3 \mathrm{H}$, $\left.\mathrm{CH}_{3}, J=7.0 \mathrm{~Hz}\right), 1.23(\mathrm{~d}, 3 \mathrm{H}, \mathrm{H}-1, J=6.2 \mathrm{~Hz}), 1.95(\mathrm{~d}, 1 \mathrm{H}, \mathrm{OH}$, $J=5.0 \mathrm{~Hz}), 2.47(\mathrm{dq}, 1 \mathrm{H}, \mathrm{H}-3, J=7.0,6.0 \mathrm{~Hz}), 3.60(\mathrm{~m}, 1 \mathrm{H}, \mathrm{H}-2)$. ${ }^{13} \mathrm{C} \mathrm{NMR}\left(62.5 \mathrm{MHz}, \mathrm{CDCl}_{3}\right): \delta=0.11$ (TMS), $17.08\left(\mathrm{CH}_{3}\right), 22.66$ (C-1), 35.80 (C-3), 70.50 (C-2), 87.48 (C-5), 107.65 (C-4). GCMS: $\mathrm{m} / \mathrm{z}=153(1)[\mathrm{M}-\mathrm{OH}]^{+}, 126(35), 117(15), 111(24), 97$ (18), 73 (100) $[\mathrm{TMS}]^{+}$. Anal. Calcd for $\mathrm{C}_{9} \mathrm{H}_{18} \mathrm{OSi}$ : C, 63.47; H, 10.65 . Found: C, 62.25; H, 9.43.

\section{erythro-3-Methyl-5-trimethylsilylpent-4-yn-2-ol}

${ }^{1} \mathrm{H}$ NMR (250 MHz, $\left.\mathrm{CDCl}_{3}\right): \delta=0.14$ (s, $\left.9 \mathrm{H}, \mathrm{TMS}\right), 1.14(\mathrm{~d}, 3 \mathrm{H}$, $\left.\mathrm{CH}_{3}, J=7.0 \mathrm{~Hz}\right), 1.23$ (d, $\left.3 \mathrm{H}, \mathrm{H}-1, J=6.2 \mathrm{~Hz}\right), 2.18$ (d, $1 \mathrm{H}, \mathrm{OH}$, $J=5.0 \mathrm{~Hz}), 2.59(\mathrm{dq}, 1 \mathrm{H}, \mathrm{H}-3, J=7.0,5.5 \mathrm{~Hz}), 3.71(\mathrm{~m}, 1 \mathrm{H}, \mathrm{H}-2)$. ${ }^{13} \mathrm{C}$ NMR (62.5 MHz, $\left.\mathrm{CDCl}_{3}\right): \delta=0.11$ (TMS), $16.17\left(\mathrm{CH}_{3}\right), 19.47$ (C-1), 35.14 (C-3), 70.26 (C-2), 86.34 (C-5), 108.20 (C-4). GCMS: identical with the threo-compound. Anal. Calcd for $\mathrm{C}_{9} \mathrm{H}_{18} \mathrm{OSi}$ : $\mathrm{C}$, 63.47; H, 10.65. Found: C, 62.53; H, 9.42.

\section{threo-1-Phenyl-2-propyl-4-trimethylsilylbut-3-yn-1-ol}

${ }^{1} \mathrm{H}$ NMR $\left(250 \mathrm{MHz}, \mathrm{CDCl}_{3}\right): \delta=0.17(\mathrm{~s}, 9 \mathrm{H}, \mathrm{TMS}), 0.86$ (t, $3 \mathrm{H}$, $\left.\mathrm{H}-3^{\prime}\right), 1.21-1.65$ (m, $4 \mathrm{H}, \mathrm{H}-1^{\prime}$ and $\left.\mathrm{H}-2^{\prime}\right), 2.62-2.79$ (m, $2 \mathrm{H}, \mathrm{H}-2$ and $\mathrm{OH}), 4.52(\mathrm{dd}, 1 \mathrm{H}, \mathrm{H}-1, J=6.6,4.0 \mathrm{~Hz}), 7.24-7.37(\mathrm{~m}, 5 \mathrm{H}$, $\mathrm{Ph}) .{ }^{13} \mathrm{C}$ NMR $\left(62.5 \mathrm{MHz}, \mathrm{CDCl}_{3}\right): \delta=0.09$ (TMS), $13.79\left(\mathrm{C}-3^{\prime}\right)$, 20.53 (C-2'), $33.24\left(\mathrm{C}-1^{\prime}\right), 42.51$ (C-2), 75.98 (C-1), 89.41 (C-4), 106.36 (C-3), 126.64 (C-4"), 127.82 (C-2" and C-6"), 128.19 (C-3" and C-5"), $141.73\left(\mathrm{C}-1^{\prime \prime}\right)$. GCMS: $m / z(\%)=139(100), 73(12)$ [TMS] $]^{+}$IR (hexane): $2167(\mathrm{C} \equiv \mathrm{C}) \mathrm{cm}^{-1}$. Anal. Calcd for $\mathrm{C}_{16} \mathrm{H}_{24} \mathrm{OSi}$ : C, 73.79; H, 9.29. Found: C, 73.20; H, 8.66.

\section{erythro-1-Phenyl-2-propyl-4-trimethylsilylbut-3-yn-1-ol}

${ }^{1} \mathrm{H}$ NMR $\left(250 \mathrm{MHz}, \mathrm{CDCl}_{3}\right): \delta=0.12$ (s, $\left.9 \mathrm{H}, \mathrm{TMS}\right), 0.89$ (t, $3 \mathrm{H}$, $\left.\mathrm{H}-3^{\prime}, J=7.0 \mathrm{~Hz}\right), 1.26-1.71\left(\mathrm{~m}, 4 \mathrm{H}, \mathrm{H}-1^{\prime}\right.$ and $\left.\mathrm{H}-2^{\prime}\right), 2.38$ (d, $1 \mathrm{H}$, $\mathrm{OH}, J=4.0 \mathrm{~Hz}), 2.76-2.85(\mathrm{~m}, 1 \mathrm{H}, \mathrm{H}-2), 4.52$ (dd, $1 \mathrm{H}, \mathrm{H}-1$, $J=5.0,4.0 \mathrm{~Hz}), 7.26-7.41(\mathrm{~m}, 5 \mathrm{H}, \mathrm{Ph}) .{ }^{13} \mathrm{C} \mathrm{NMR}(62.5 \mathrm{MHz}$, $\left.\mathrm{CDCl}_{3}\right): \delta=0.03$ (TMS), $13.85\left(\mathrm{C}-3^{\prime}\right), 20.52\left(\mathrm{C}-2^{\prime}\right), 31.86\left(\mathrm{C}-1^{\prime}\right)$, 41.24 (C-2), 75.63 (C-1), 89.23 (C-4), 106.94 (C-3), 126.66 (C-4"), 127.59 (C-2" and C-6"), 128.89 (C-3" and C-5"), 141.55 (C-1"). GCMS: identical to the threo-compound. IR (hexane): $2169(\mathrm{C} \equiv \mathrm{C})$ $\mathrm{cm}^{-1}$. Anal. Calcd for $\mathrm{C}_{16} \mathrm{H}_{24} \mathrm{OSi}$ : C, 73.79; H, 9.29. Found: $\mathrm{C}$, 73.07; H, 9.97. 
threo-1-(4-Methoxyphenyl)-2-propyl-4-trimethylsilyl-

but-3-yn-1-ol

${ }^{1} \mathrm{H}$ NMR $\left(250 \mathrm{MHz}, \mathrm{CDCl}_{3}\right): \delta=0.19$ (s, $\left.9 \mathrm{H}, \mathrm{TMS}\right), 0.87$ (t, $3 \mathrm{H}$, $\left.\mathrm{H}-3^{\prime}, J=7.0 \mathrm{~Hz}\right), 1.19-1.62\left(\mathrm{~m}, 5 \mathrm{H}, \mathrm{H}^{\prime} 1^{\prime}, \mathrm{H}-2^{\prime}\right.$ and $\left.\mathrm{OH}\right), 2.66-2.74$ (m, $1 \mathrm{H}, \mathrm{H}-2), 3.82\left(\mathrm{~s}, 3 \mathrm{H}, \mathrm{OCH}_{3}\right), 4.63(\mathrm{~m}, 1 \mathrm{H}, \mathrm{H}-1), 6.89$ (d, 2 $\mathrm{H}, \mathrm{H}-3^{\prime \prime}$ and $\mathrm{H}-5^{\prime \prime}, J=9.3 \mathrm{~Hz}$ ), 7.29 (d, $2 \mathrm{H}, \mathrm{H}-2^{\prime \prime}$ and $\mathrm{H}-6^{\prime \prime}, J=9.3$ $\mathrm{Hz}) .{ }^{13} \mathrm{C}$ NMR $\left(62.5 \mathrm{MHz}, \mathrm{CDCl}_{3}\right): \delta=0.12$ (-TMS), $13.80\left(\mathrm{C}-3^{\prime}\right)$, $20.50\left(\mathrm{C}-2^{\prime}\right), 33.18\left(\mathrm{C}-1^{\prime}\right), 42.52(\mathrm{C}-2), 55.24\left(\mathrm{OCH}_{3}\right), 75.70(\mathrm{C}-1)$, 89.23 (C-4), 106.67 (C-3), 113.63 (C-3" and C-5"), 127.84 (C-2" and C-6"), $133.81\left(\mathrm{C}-1^{\prime \prime}\right), 159.26\left(\mathrm{C}-4^{\prime \prime}\right)$. GCMS: $m / z(\%)=209$ (15), 73 (100) [TMS $]^{+}$. IR (hexane): $2167(\mathrm{C} \equiv \mathrm{C}) \mathrm{cm}^{-1}$. Anal. Calcd for $\mathrm{C}_{17} \mathrm{H}_{26} \mathrm{O}_{2} \mathrm{Si}$ : C, 70.29; H, 9.02. Found: C, 72.61; H, 8.87.

erythro-1-(4-Methoxyphenyl)-2-propyl-4-trimethylsilylbut-3-yn-1-ol

${ }^{1} \mathrm{H}$ NMR (250 MHz, CDCl $)_{3}$ ): $\delta=0.14$ (s, $\left.9 \mathrm{H}, \mathrm{TMS}\right), 0.88$ (t, $3 \mathrm{H}$, $\left.\mathrm{H}-3^{\prime}, J=7.0 \mathrm{~Hz}\right), 1.21-1.70\left(\mathrm{~m}, 4 \mathrm{H}, \mathrm{H}-1^{\prime}\right.$ and $\left.\mathrm{H}-2^{\prime}\right), 2.23(\mathrm{~d}, 1 \mathrm{H}$, $\mathrm{OH}, J=6.8 \mathrm{~Hz}), 2.77-2.85(\mathrm{~m}, 1 \mathrm{H}, \mathrm{H}-2), 3.83\left(\mathrm{~s}, 3 \mathrm{H}, \mathrm{OCH}_{3}\right), 4.68$ (t, $1 \mathrm{H}, \mathrm{H}-1, J=4.3 \mathrm{~Hz}), 6.88\left(\mathrm{~d}, 2 \mathrm{H}, \mathrm{H}-3^{\prime \prime}\right.$ and $\left.\mathrm{H}-5^{\prime \prime}, J=9.3 \mathrm{~Hz}\right)$, 7.32 (d, $2 \mathrm{H}, \mathrm{H}-2^{\prime \prime}$ and $\left.\mathrm{H}-6{ }^{\prime \prime}, J=9.3 \mathrm{~Hz}\right) .{ }^{13} \mathrm{C}$ NMR $(62.5 \mathrm{MHz}$, $\left.\mathrm{CDCl}_{3}\right): \delta=0.06$ (TMS), $13.89\left(\mathrm{C}-3^{\prime}\right), 20.53\left(\mathrm{C}-2^{\prime}\right), 32.05\left(\mathrm{C}-1^{\prime}\right)$ $41.31(\mathrm{C}-2), 55.26\left(-\mathrm{OCH}_{3}\right), 75.24(\mathrm{C}-1), 88.58(\mathrm{C}-4), 107.03(\mathrm{C}-3)$, 113.32 (C-3" and C-5"), 127.83 (C-2" and C-6"), 133.81 (C-1"), 159.11 (C-4"). GCMS: identical to the threo-compound. IR (hexane): $2169(\mathrm{C} \equiv \mathrm{C}) \mathrm{cm}^{-1}$. Anal. Calcd for $\mathrm{C}_{17} \mathrm{H}_{26} \mathrm{O}_{2} \mathrm{Si}: \mathrm{C}, 70.29$; $\mathrm{H}$, 9.02. Found: $\mathrm{C}, 71.81 ; \mathrm{H}, 8.91$.

\section{threo-1-Cyclohexyl-2-propyl-4-trimethylsilylbut-3-yn-1-ol} ${ }^{1} \mathrm{H} \mathrm{NMR}\left(250 \mathrm{MHz}, \mathrm{CDCl}_{3}\right): \delta=0.15(\mathrm{~s}, 9 \mathrm{H}, \mathrm{TMS}), 0.87-2.00(\mathrm{~m}$, $19 \mathrm{H}$, cyclohexyl, H-1', H-2', H-3' and $\mathrm{OH}), 2.62$ (m, $1 \mathrm{H}, \mathrm{H}-2)$, $3.10(\mathrm{~m}, 1 \mathrm{H}, \mathrm{H}-1) .{ }^{13} \mathrm{C} \mathrm{NMR}\left(62.5 \mathrm{MHz}, \mathrm{CDCl}_{3}\right): \delta=0.17$ (TMS), $13.88\left(\mathrm{C}-3^{\prime}\right), 20.04\left(\mathrm{C}-2^{\prime}\right), 26.43\left(\mathrm{C}-4^{\prime \prime}\right), 26.7\left(\mathrm{C}-3^{\prime \prime}\right.$ and $\left.\mathrm{C}-5^{\prime \prime}\right)$, $28.35\left(\mathrm{C}-2^{\prime \prime}\right), 29.66\left(\mathrm{C}-6^{\prime \prime}\right), 34.31\left(\mathrm{C}-1^{\prime}\right), 36.84(\mathrm{C}-2), 42.13\left(\mathrm{C}-1^{\prime \prime}\right)$, 78.14 (C-1), 88.64 (C-4), 106.59 (C-3). GCMS: $m / z(\%)=266$ $\left.(<1.0)\left[\mathrm{M}^{+}\right], 265(<1.0)[\mathrm{M}-\mathrm{H}]^{+}, 248(<1.0)\left[\mathrm{M}-\mathrm{H}_{2} \mathrm{O}\right]^{+}\right), 233$ (<1.0), 185 (29.0), 154 (53), 73 (100.0) [TMS] ${ }^{+}$. IR (hexane): 2167 $(\mathrm{C} \equiv \mathrm{C}) \mathrm{cm}^{-1}$. Anal. Calcd for $\mathrm{C}_{16} \mathrm{H}_{30} \mathrm{OSi}: \mathrm{C}, 72.11 ; \mathrm{H}, 11.34$. Found: C, $71.87 ; \mathrm{H}, 11.17$.

\section{erythro-1-Cyclohexyl-2-propyl-4-trimethylsilylbut-3-yn-1-ol}

${ }^{1} \mathrm{H}$ NMR $\left(250 \mathrm{MHz}, \mathrm{CDCl}_{3}\right): \delta=0.14$ (s, $\left.9 \mathrm{H}, \mathrm{TMS}\right), 0.88-2.00(\mathrm{~m}$, $19 \mathrm{H}$, cyclohexyl, H-1', H-2', H-3' and OH), 2.50-2.60 (m, $1 \mathrm{H}, \mathrm{H}-$ 2), $3.36(\mathrm{t}, 1 \mathrm{H}, \mathrm{H}-1, J=6.0 \mathrm{~Hz}) .{ }^{13} \mathrm{C}$ NMR $\left(62.5 \mathrm{MHz}, \mathrm{CDCl}_{3}\right)$ : $\delta=0.16(-\mathrm{TMS}), 13.99\left(\mathrm{C}-3^{\prime}\right), 20.64\left(\mathrm{C}-2^{\prime}\right), 26.07\left(\mathrm{C}-4^{\prime \prime}\right), 26.34(\mathrm{C}-$ $3^{\prime \prime}$ and C-5"), $27.51\left(\mathrm{C}-2^{\prime \prime}\right), 29.72\left(\mathrm{C}-6^{\prime \prime}\right), 31.05\left(\mathrm{C}-1^{\prime}\right), 36.88(\mathrm{C}-2)$, 40.12 (C-1"), 78.01 (C-1), 87.56 (C-4), 108.22 (C-3). GCMS: identical to the threo-compound. IR (hexane): $2167(\mathrm{C} \equiv \mathrm{C}) \mathrm{cm}^{-1}$. Anal. Calcd for $\mathrm{C}_{16} \mathrm{H}_{30} \mathrm{OSi}$ C, $72.11 ; \mathrm{H}, 11.34$. Found: C, 71.78; H, 10.89 .

threo-2,2-Dimethyl-4-propyl-6-trimethylsilylhex-5-yn-3-ol ${ }^{1} \mathrm{H}$ NMR $\left(250 \mathrm{MHz}, \mathrm{CDCl}_{3}\right): \delta=0.18$ (s, $\left.9 \mathrm{H}, \mathrm{TMS}\right), 0.92(\mathrm{t}, 3 \mathrm{H}$, $\left.\mathrm{H}-3^{\prime}, J=7.0 \mathrm{~Hz}\right), 0.94(\mathrm{~s}, 9 \mathrm{H},-t-\mathrm{Bu}), 1.24-1.79\left(\mathrm{~m}, 4 \mathrm{H}, \mathrm{H}-1^{\prime}\right.$ and H-2'), 2.09 (d, $1 \mathrm{H}, \mathrm{OH}, J=10.7 \mathrm{~Hz}), 2.68(\mathrm{~m}, 1 \mathrm{H}, \mathrm{H}-2), 3.06(\mathrm{dd}$, $1 \mathrm{H}, \mathrm{H}-1, J=10.7,1.2 \mathrm{~Hz}) \cdot{ }^{13} \mathrm{C}$ NMR $\left(62.5 \mathrm{MHz}, \mathrm{CDCl}_{3}\right): \delta=$ -0.02 (TMS), 13.79 (C-3'), 20.58 (C-2'), $26.32(\mathrm{C}-1$ and $2 \times \mathrm{C} 2-$ $\left.\mathrm{CH}_{3}\right), 34.87(\mathrm{C}-2), 36.12\left(\mathrm{C}-1^{\prime}\right), 37.14(\mathrm{C}-4), 79.74(\mathrm{C}-3), 90.66(\mathrm{C}-$ 6), $106.41(\mathrm{C}-5)$. GCMS: $m / z(\%)=154$ (7) $\left[\mathrm{M}-t \text {-Bu, } \mathrm{C}_{2} \mathrm{H}_{5}\right]^{+}, 73$ (100) $[\mathrm{TMS}]^{+}$. IR (hexane): $2166(\mathrm{C} \equiv \mathrm{C}) \mathrm{cm}^{-1}$. Anal. Calcd for $\mathrm{C}_{14} \mathrm{H}_{28} \mathrm{OSi}$ C, 69.93; H, 11.73. Found: C, 69.13; H, 11.10.

\section{threo-3-Propyl-5-trimethylsilylpent-4-yn-2-ol}

${ }^{1} \mathrm{H}$ NMR $\left(250 \mathrm{MHz}, \mathrm{CDCl}_{3}\right): \delta=0.16(\mathrm{~s}, 9 \mathrm{H}, \mathrm{TMS}), 0.93(\mathrm{t}, 3 \mathrm{H}$, H-3' $J=7.4 \mathrm{~Hz}), 1.24$ (d, $3 \mathrm{H}, \mathrm{H}-1, J=6.0 \mathrm{~Hz}), 1.30-1.65(\mathrm{~m}, 4 \mathrm{H}$,

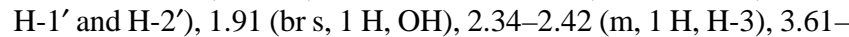
$3.71(\mathrm{~m}, 1 \mathrm{H}, \mathrm{H}-2) .{ }^{13} \mathrm{C} \mathrm{NMR}\left(62.5 \mathrm{MHz}, \mathrm{CDCl}_{3}\right): \delta=0.17$ (TMS), 13.91(C-3'), 20.67 (C-2'), 21.25 (C-1), $33.51\left(\mathrm{C}-1^{\prime}\right), 41.74(\mathrm{C}-3)$, 69.19 (C-2), 88.70 (C-5), 106.45 (C-4). GCMS: $m / z(\%)=154(25)$
$[\mathrm{M}-\mathrm{Et}, \mathrm{Me}]^{+}, 73$ (100) $[\mathrm{TMS}]^{+}$. IR (hexane): $2166(\mathrm{C} \equiv \mathrm{C}) \mathrm{cm}^{-1}$. Anal. Calcd for $\mathrm{C}_{12} \mathrm{H}_{24} \mathrm{OSi}$ : C, 66.60; H, 11.18. Found: $\mathrm{C}, 66.46 ; \mathrm{H}$, 10.86.

erythro-3-Propyl-5-trimethylsilylpent-4-yn-2-ol

${ }^{1} \mathrm{H}$ NMR $\left(250 \mathrm{MHz}, \mathrm{CDCl}_{3}\right): \delta=0.14(\mathrm{~s}, 9 \mathrm{H}, \mathrm{TMS}), 0.92(\mathrm{t}, 3 \mathrm{H}$, C-3', $J=7.0 \mathrm{~Hz}), 1.24(\mathrm{~d}, 3 \mathrm{H}, \mathrm{H}-1, J=6.0 \mathrm{~Hz}), 1.28-1.62(\mathrm{~m}, 4 \mathrm{H}$, $\mathrm{H}-1^{\prime}, \mathrm{H}-2^{\prime}$ and $\left.\mathrm{OH}\right), 2.49-2.58(\mathrm{~m}, 1 \mathrm{H}, \mathrm{H}-3), 3.67-3.80(\mathrm{~m}, 1 \mathrm{H}, \mathrm{H}-$ 2). ${ }^{13} \mathrm{C}$ NMR (62.5 MHz, $\left.\mathrm{CDCl}_{3}\right): \delta=0.11$ (TMS), $16.17\left(\mathrm{C}-3^{\prime}\right)$, 19.47 (C-2'), 20.83 (C-1), 35.14 (C-1'), 41.42 (C-3), 70.26 (C-2), 86.34 (C-5), 108.20 (C-4). GCMS: identical to the threo-compound. IR (hexane): $2167(\mathrm{C} \equiv \mathrm{C}) \mathrm{cm}^{-1}$. Anal. Calcd for $\mathrm{C}_{12} \mathrm{H}_{24} \mathrm{OSi}$ : $\mathrm{C}$, $66.60 ; \mathrm{H}, 11.18$. Found: C, 66.03; H, 10.66.

\section{Acknowledgment}

This work was supported by the EU Commission, Directorate General XII. We are thankful to the Merck KGaA and the Wacker AG for the generous donation of reagents.

\section{References and Notes}

(1) Lanthanides in Organic Synthesis, part 6. For part 5, see: Fischer, S.; Groth, U.; Jeske, M.; Schütz, T. Synlett 2002, 1922.

(2) For reviews, see: (a) Moreau, J. L. In The Chemistry of Ketenes, Allenes, and Related Compounds, Part 1; Patai, S., Ed.; Wiley-VCH: New York, 1980, 363-413.

(b) Yamamoto, H. In Comprehensive Organic Synthesis, Vol. 2; Trost, B. M.; Fleming, I.; Heatchcock, C. H., Eds.; Pergamon Press: Oxford, 1991, 81-98. (c) Marshall, J. A.; Gung, B. W.; Grachan, M. L. In Modern Allene Chemistry; Krause, N.; Hashmi, A. S. K., Eds.; Wiley-VCH: Weinheim, 2004, 493-592.

(3) (a) Zweifel, G.; Hahn, G. J. Org. Chem. 1984, 49, 4565. (b) Trost, B. M.; Rhee, Y. H. J. Am. Chem. Soc. 1999, 121, 11680.

(4) Marshall, J. A.; Xie, S. J. Org. Chem. 1995, 60, 7230.

(5) (a) Ishiguro, M.; Ikeda, N.; Yamamoto, H. J. Org. Chem. 1982, 47, 2225. (b) Kobayashi, S.; Nishio, K. J. Am. Chem. Soc. 1995, 117, 6392. (c) Kurono, N.; Sugita, K.; Tokuda, M. Tetrahedron 2000, 56, 847.

(6) Furuta, K.; Ishiguro, M.; Haruta, R.; Ikeda, N.; Yamamoto, H. Bull. Chem. Soc. Jpn. 1984, 57, 2768.

(7) Groth, U.; Richter, N.; Kalogerakis, A. Synlett 2006, 905.

(8) Eckenberg, P.; Groth, U.; Köhler, T. Liebigs Ann. Chem. 1994, 673.

(9) For reviews, see: (a) Imamoto, T. In Comprehensive Organic Chemistry, Vol. 1; Trost, B. M.; Fleming, I.; Schreiber, S. L., Eds.; Pergamon Press: Oxford, 1991, 231249. (b) Molander, G. A. Chem. Rev. 1992, 92, 29. (c) Imamoto, T. In Lanthanides in Organic Chemistry; Academic Press: New York, 1994, 81-95.

(10) 3-Bromo-1-(trimethylsilyl)but-1-yne (1) was synthesized in analogy to a procedure for the preparation of 3-bromo-1(trimethylsilyl)prop-1-yne: Wu, R.; Schramm, J. S.; Pearson, D. L.; Tour, J. M. J. Org. Chem. 1996, 61, 6906.

(11) 3-Bromo-1-(trimethylsilyl)hex-1-yne (2) was prepared by addition of lithium(trimethylsilyl)acetylide to butyraldehyde, tosylation of the resulting alcohol and nucleophilic substitution of the tosylate by bromide under Finkelstein conditions. See: (a) Wenkert, E.; Leftin, M. H.; Michelotti, E. J. Org. Chem. 1985, 50, 1122. (b) Wipf, P.; Aoyama, Y.; Benedum, T. Org. Lett. 2004, 6, 3593. (c) Darresh, S.; Grant, A. S.; Magee, D. I.; Valenta, Z. Can. J. Chem. 1991, 69, 712. 